\title{
Pulmonary vein isolation and the Cox maze procedure only partially denervate the atrium
}

\author{
Shelly C. Lall, MD, Kelley V. Foyil, MS, Shun-Ichiro Sakamoto, MD, Rochus K. Voeller, MD, John P. Boineau, MD, \\ Ralph J. Damiano, Jr, MD, and Richard B. Schuessler, PhD
}

From the Division of Cardiothoracic Surgery, Washington University School of Medicine, Saint Louis, Mo.

Ralph Damiano reports fees from Atricure, Edwards, Medtronic, and Medical CV and grant support from Atricure and Estech. Richard Schuessler reports fees from Atricure and Medtronic and grant support from Atricure and Estech.

Supported in part by National Institutes of Health grants RO1-HL032257 and T32HL007776.

Read at the Thirty-third Meeting of the Western Thoracic Surgical Association, Santa Ana Pueblo, NM, June 27-30, 2007.

Received for publication June 21, 2007; revisions received Sept 20, 2007; accepted for publication Nov 26, 2007.

Address for reprints: Richard B. Schuessler, $\mathrm{PhD}$, Washington University School of Medicine, 660 South Euclid Ave, Box 8234, Saint Louis, MO 63110 (E-mail: schuesslerr@wustl.edu).

J Thorac Cardiovasc Surg 2008;135:894900

$0022-5223 / \$ 34.00$

Copyright $(\subset) 2008$ by The American Association for Thoracic Surgery

doi:10.1016/j.jtcvs.2007.11.044
Objectives: The effects of ablation lines on myocardial innervation and response to autonomic stimuli are unclear. This study examined the effects of radiofrequency ablation on atrial autonomic innervation and compared pulmonary vein isolation and the biatrial Cox maze procedure.

Methods: In 12 acute canines right and left vagosympathetic trunks and right and left stellate ganglia were isolated. Each nerve was stimulated before bipolar ablation, after pulmonary vein isolation, and after the Cox maze procedure. Nadolol $(n=6)$ and atropine $(\mathrm{n}=6)$ were administered to block sympathetic and parasympathetic responses, respectively. Changes in heart rate and atrioventricular interval were compared. Changes in QRST area relative to an isoelectric baseline (index of local innervation) were calculated.

Results: Sympathetic stimulation of each nerve and parasympathetic stimulation of the vagosympathetic trunks caused significant changes in heart rate and atrioventricular interval. After pulmonary vein isolation, the effect of $33 \%$ of the nerves on heart rate changes was eliminated. The Cox maze procedure eliminated right stellate sympathetic effects on heart rate. Fifty percent of the nerves caused heart rate changes after the Cox maze procedure. There was no significant effect of either lesion set on atrioventricular interval changes. Stimulation of $50 \%$ of nerves after pulmonary vein isolation produced local area changes significantly different from control area. After the Cox maze procedure, a different $50 \%$ of the nerves produced local changes different from those seen after pulmonary vein isolation.

Conclusions: Surgical ablation procedures disrupted innervation, affecting heart rate but not atrioventricular interval. Autonomic innervation affecting the atria was changed by pulmonary vein isolation and additionally by the Cox maze procedure. Residual autonomic effects were present even after the complete Cox maze procedure.

$\mathrm{T}$ The Cox maze $(\mathrm{CM})$ procedure is considered the gold standard for curing atrial fibrillation (AF). ${ }^{1,2}$ The cut-and-sew CM III procedure, introduced in 1987, is technically demanding and not widely performed by cardiac surgeons. Bipolar radiofrequency ablation (RFA) is one of several energy sources used to replace some of the incisions of the CM III procedure, simplifying and shortening the procedure with equivalent short-term efficacy and 1-year freedom from AF (CM IV procedure) ${ }^{3,4}$ Pulmonary vein isolation (PVI), instead of a complete CM IV procedure, has also been used as a surgical treatment of $\mathrm{AF}^{5,6}$

Autonomic nerves affect both the sinus and atrioventricular (AV) nodes and the repolarization phase of the cardiac action potential. ${ }^{7-9}$ Recently, animal and human studies have suggested that autonomic tone plays an important role in the initiation and maintenance of AF. ${ }^{10,11}$ As a result, it has been the suggested that denervation of the atria might be a therapeutic option for the treatment of AF. It has been shown that the cut-and-sew CM III procedure produces partial denervation of the heart. ${ }^{12-14}$ In a canine model this procedure has been shown to produce autonomic denervation of a significant portion of both atria and the atrial septum. ${ }^{15}$ In human subjects who have 

Abbreviations and Acronyms
$\mathrm{AF}=$ atrial fibrillation
$\mathrm{AV}=$ atrioventricular
$\mathrm{CM}=$ Cox maze
LVST $=$ left cervical vagosympathetic trunk
PVI = pulmonary vein isolation
RFA = radiofrequency ablation
RVST $=$ right cervical vagosympathetic trunk

had the classic CM III procedure, loss of heart rate variability suggests the loss of most autonomic input to the sinus node. ${ }^{16}$ Therefore it is important to know whether the use of bipolar RFA used in the CM IV and PVI procedures completely or partially denervates the atria.

The aim of this study was to examine the effect that bipolar radiofrequency lesions used in PVI and the CM procedure have on sympathetic and parasympathetic innervation of the atria.

\section{Materials and Methods}

All animals received humane care in compliance with the "Guide for the care and use of laboratory animals," published by the National Research Council (National Academy Press, 1996). The study protocol was approved by the Animal Studies Committee at Washington University School of Medicine.

Canines were chosen because they have an innervation to the atria that is similar to that of human subjects. ${ }^{17,18}$ Each animal was anesthetized with intravenous propofol $(5-7 \mathrm{mg} / \mathrm{kg})$, intubated with a cuffed endotracheal tube, and ventilated with a pressure-controlled ventilator, titrating inhaled isoflurane (1\% to $3 \%$ ) during the procedure. Heart rate, respiratory rate, pulse oximetry, temperature, and limb-lead electrocardiographic values were continuously monitored. The femoral artery and vein were accessed and used for blood draws and continuous pressure monitoring. Serum electrolyte concentration, $\mathrm{pH}$, and arterial oxygen tension were monitored and normalized.

A median sternotomy was performed. The azygous vein was identified and ligated. The right and left cervical vagosympathetic trunks (RVST and LVST) and right and left stellate ganglia were decentralized. The pericardium was opened, and 3 electrode templates containing 252 unipolar electrodes were sutured on the right and left atria.

\section{Electrophysiologic Data Collection}

Twelve mongrel canines were randomly assigned to one of 2 groups: one received propranolol $(0.2 \mathrm{mg} / \mathrm{kg})$ or nadolol $(1 \mathrm{mg} /$ $\mathrm{kg})$, and the other received atropine $(0.08 \mathrm{mg} / \mathrm{kg}$ bolus and then $0.04 \mathrm{mg} / \mathrm{kg}$ every hour) before the collection of baseline electrophysiologic data. Nerves were stimulated with the use of a pulse generator (Grass Instruments, Quincy, Mass) with a frequency of $10 \mathrm{~Hz}$, amplitude of $10 \mathrm{~V}$, and a 5-ms pulse width duration. A bipolar pacing electrode was placed on the right atrial appendage to control rate. The tissue was paced at $300 \mathrm{~ms}$, a 2-ms duration, and twice the pacing threshold.

The right and left atria were mapped with custom-made electrode templates containing 252 unipolar electrodes. The electrode templates were constructed from a form-fitting silicon elastomer (Specialty Silicone Fabricators, Paso Robles, Calif) that fit snugly on the entire atrial epicardium and contained 0.5-mm-diameter electrodes (Pacific Wire \& Cable, Inc, Santa Ana, Calif). The interelectrode distance between the points was $5 \mathrm{~mm}$. Atrial electrograms were recorded during (1) spontaneous normal sinus rhythm, (2) continuous pacing, (3) continuous pacing with nerve stimulation, and (4) spontaneous normal sinus rhythm with stimulation. This was repeated for each of the 4 nerves (RVST, right stellate, LVST, and left stellate). Data were acquired with a PC-based data acquisition and analysis system. The system included custom-programmed software capable of data acquisition, management, display, and analysis. Unipolar electrograms were recorded at a gain of 125 with a frequency response of 0.5 to $1000 \mathrm{~Hz}$. Each channel was digitized at $2000 \mathrm{~Hz}$ with 12-bit resolution. The electrode templates were approximated to the atria by using sutures passed through the tissue and template and then secured with a snare. This allowed the temporary removal and replacement of the templates in exactly the same location.

\section{Surgical Procedure}

The modified CM procedure was performed without cardiopulmonary bypass and included nearly all the right and left atrial lesions of the traditional procedure (Figure 1). ${ }^{19}$ The heart was manipulated with the aid of a suction retractor (AXIUS Xpose 3; Guidant Corp, Santa Clara, Calif). Blunt dissection was used to allow umbilical tape to be passed around the right pulmonary veins. The Isolator LP bipolar device (Atricure, Inc, Cincinnati, Ohio) was then passed around the right pulmonary veins, and a cuff of surrounding atrial tissue was clamped and ablated on each side; the same was done around the left pulmonary veins. After completion of the PVI, electrophysiologic data, as described above, were obtained.

A purse-string suture was placed midway between the confluence of the superior vena cava and inferior vena cava. The radiofrequency device was introduced through this purse-string suture. Superior and inferior vena caval lesions were created. The snare was relaxed, and the device was repositioned to the AV groove. At this location, the right coronary artery was dissected free, allowing the tip of the device to pass beneath it.

The radiofrequency device was used to circumferentially ablate the left atrial appendage on the epicardial surface. The device was introduced through a purse-string suture at the base of the left atrial

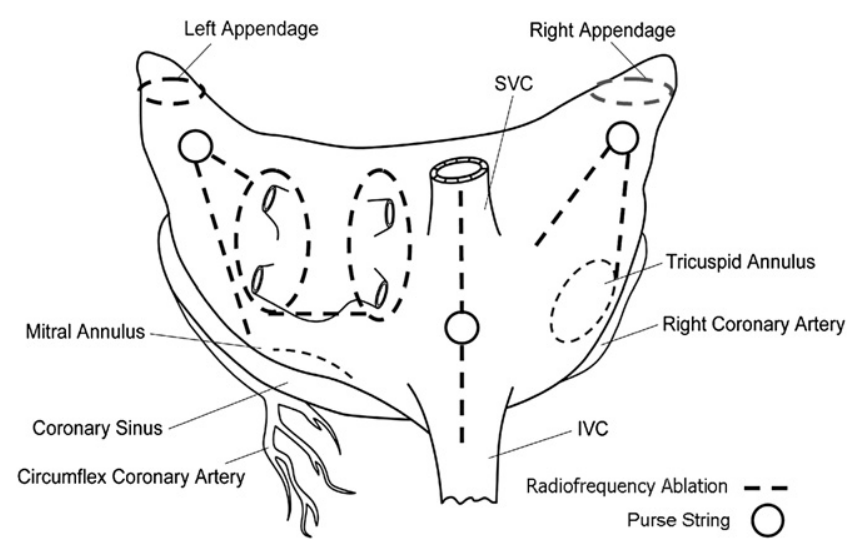

Figure 1. Modified Cox maze lesion set. SVC, Superior vena cava; $I V C$, inferior vena cava. 


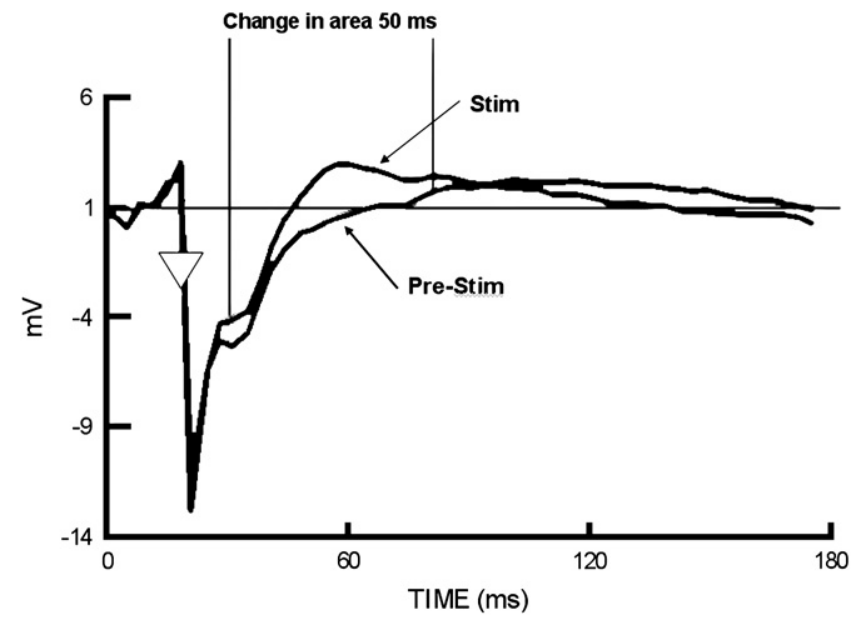

Figure 2. Calculation of change in QRST area for unipolar electrographic analysis. Stim, Stimulation.

appendage and positioned with the tip directed into the left superior pulmonary vein to create the connecting lesion from the left atrial appendage to the left pulmonary vein. It was then redirected to create the connecting lesion between the left atrial appendage and mitral valve. The left circumflex artery was dissected free so that the tip of the clamp could pass beneath the artery.

A purse-string suture was placed at the right atrial appendage, and the radiofrequency device was positioned to create the right atrial free wall lesion. The device was then placed with the tip at the AV groove. The right coronary artery was dissected at this point so that the tip of the device could pass beneath the coronary artery. Additionally, the right atrial appendage was circumferentially ablated.
The heart was repositioned to expose the intra-atrial groove. The radiofrequency device was placed epicardially posterior to the inferior vena cava, with tissue from the right inferior pulmonary vein to the left inferior pulmonary vein within the jaws of the clamp to create a connecting lesion between the inferior aspects of the pulmonary veins. The interposing tissue was ablated to complete the connecting lesion between the right and left pulmonary veins. After completing these remaining lesions, the electrode templates were reattached, and electrophysiologic data were acquired.

\section{Data Analysis}

Spontaneous normal sinus rhythm cycle lengths before and during stimulation were measured, and the change in heart rate was calculated. Likewise, the AV interval was calculated by using data obtained during continuous pacing.

The local activation time was defined as the time of the maximum negative derivative of the unipolar electrogram. All electrograms were edited visually to verify accuracy of the computerselected activation times. Previously, QRST area measurements of local electrograms have been used to detect changes in atrial electrical activity induced by stimulation of individual nerves. ${ }^{20,21}$ The change in area of QRST deflection in the unipolar waveform is a measure of change of action potential duration, which is sensitive to sympathetic and parasympathetic stimulation. ${ }^{22}$ QRST area was determined by using an isoelectric diastolic baseline calculated just after the pacing spike. The area calculation started $10 \mathrm{~ms}$ after the local activation and proceeded for $20 \mathrm{~ms}$ with sympathetic stimulation and $50 \mathrm{~ms}$ with parasympathetic stimulation (Figure 2). The absolute value of the difference in the area under the curve in the paced data and paced plus stimulation data was calculated and normalized to the paced data. To determine the level of noise in the data (used as control value), 2 successive beats of paced data without stimulation were analyzed in the same manner.
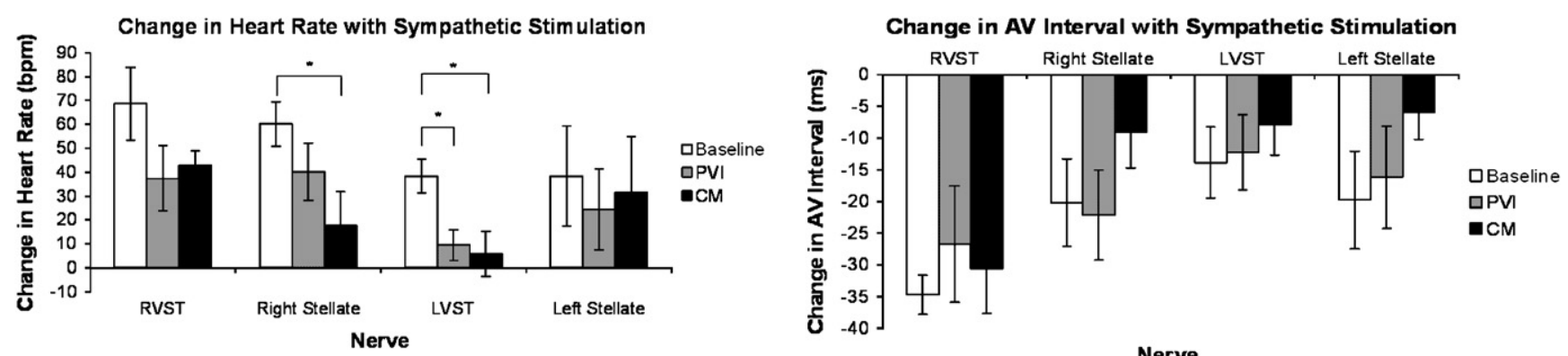

Nerve
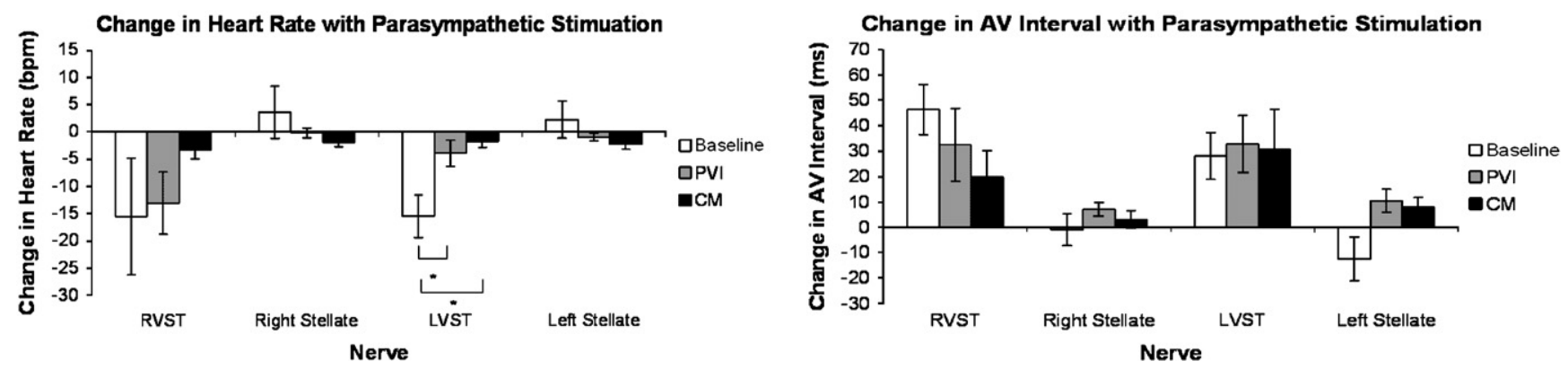

Figure 3. Change in heart rate and atrioventricular interval. ${ }^{*} \boldsymbol{P} \leq .05$. AV, Atrioventricular; $R V S T$, right vagosympathetic trunk; LVST, left vagosympathetic trunk; PVI, pulmonary vein isolation; CM, Cox maze procedure. 

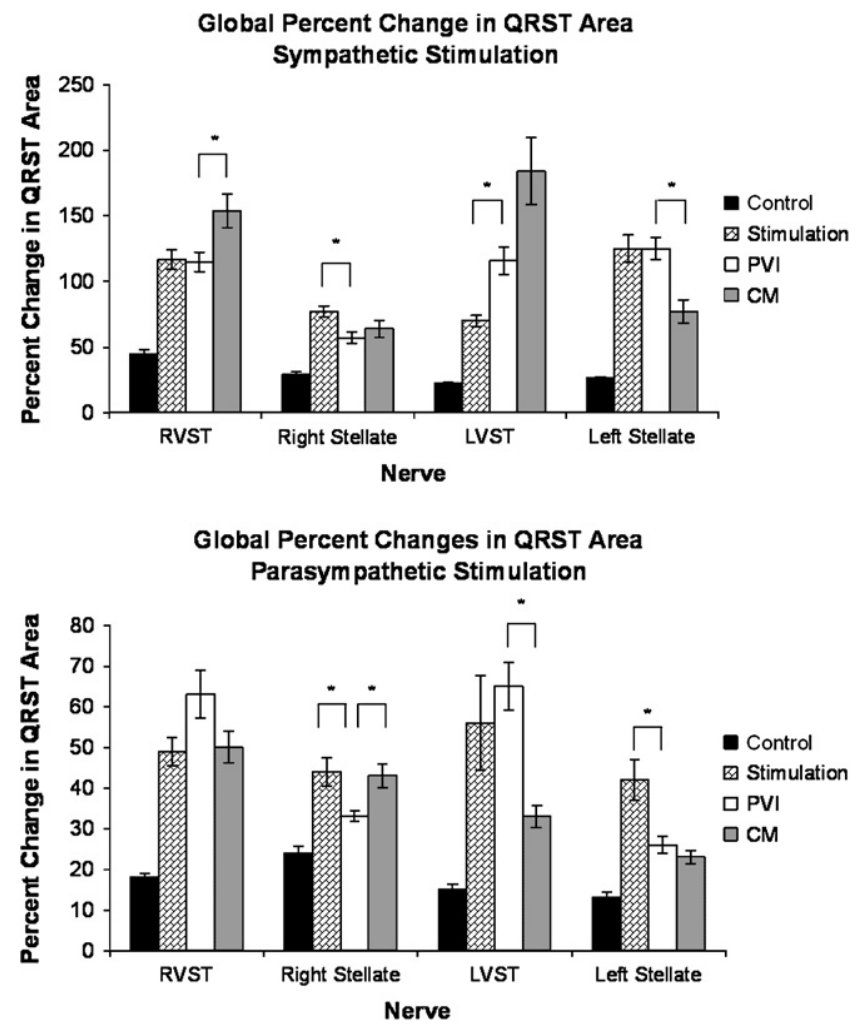

Figure 4. Global percentage change in ORST area. Values after stimulation, $\mathrm{PVI}$, and the Cox maze procedure are significantly different $(P \leq .05)$ from control values in each of the 4 nerves for both sympathetic and parasympathetic stimulation. ${ }^{*} P \leq .05$. RVST, Right vagosympathetic trunk; $L V S T$, left vagosympathetic trunk; $P V I$, pulmonary vein isolation; CM, Cox maze procedure.

Statistical analysis was provided by using repeated-measures analysis of variance procedures with one factor. Adjustments were made with the Fisher least significant difference test or pairwise for multiple comparisons with the Fisher least significant difference test. Values are presented as means \pm standard error.

\section{Results}

\section{Change in Heart Rate and AV Interval}

Sympathetic stimulation of each of the 4 nerves caused significant changes in heart rate. Heart rate changes caused by right stellate sympathetic stimulation were different than baseline values after the CM procedure ( $60 \pm 9$ vs $18 \pm 14$ beats/ $\min , P=.028$, Figure 3 ). After both PVI ( $38 \pm 7$ vs $9 \pm 6$ beats/min, $P=.022)$ and the $\mathrm{CM}$ procedure (38 \pm 7 vs $6 \pm$ 9 beats/min, $P=.012$ ), heart rate changes caused by LVST sympathetic stimulation were different than baseline values. However, there was no difference between PVI and CM changes ( $9 \pm 6$ vs $6 \pm 9$ beats/min, $P=.743$ ). Sympathetic stimulation of the RVST and left stellate did not produce significant heart rate changes between baseline, PVI, and CM.

Parasympathetic stimulation of the RVST and LVST caused changes in heart rate, whereas that of the right and left stellate did not. Heart rate changes caused by LVST parasympathetic stimulation after both PVI $(-15 \pm 4$ vs $-4 \pm 2$ beats $/ \mathrm{min}, P=.011)$ and the $\mathrm{CM}$ procedure $(-15 \pm 4 \mathrm{vs}-2$ $\pm 1 \mathrm{CM}, P=.004$ ) were different than baseline values (Figure 3). However, there was no difference between PVI and $\mathrm{CM}(-4 \pm 2$ vs. $-2 \pm 1, P=0.583)$. Parasympathetic stimulation of RVST produced heart rate changes not significantly different between baseline, PVI, and CM.

Sympathetic stimulation of each of the 4 nerves and parasympathetic stimulation of the RVST and LVST caused significant changes in the AV interval. Changes in the AV intervals measured in control, after PVI, and after the completion of the $\mathrm{CM}$ procedure were not significantly different (Figure 3 ).

\section{Change in QRST Area}

Sympathetic and parasympathetic stimulation of all 4 nerves produces global changes in area different from control values in all conditions (Figure 4). Changes after PVI were significantly different from those with stimulation alone with sympathetic stimulation of the right stellate $(77 \pm 4$ vs $57 \pm 5$, $P=.007)$ and $\operatorname{LVST}(70 \pm 4$ vs $116 \pm 10, P=0)$, as well as parasympathetic stimulation of the right stellate (44 \pm 3 vs $33 \pm 1, P=.007)$ and left stellate $(42 \pm 5$ vs $26 \pm 2$, $P=.003$ ). After the $\mathrm{CM}$ procedure, significant changes in area from those after PVI occurred with sympathetic stimulation of the RVST (115 \pm 8 vs $154 \pm 13, P=.008)$ and left stellate (125 \pm 8 vs $77 \pm 9, P=.001)$, as well as parasympathetic stimulation of the right stellate ( $33 \pm 1$ vs $43 \pm 3, P=$ $.005)$ and LVST ( $65 \pm 6$ vs $33 \pm 3, P=0)$.

Changes in area different from control values in the right atrium were observed with sympathetic and parasympathetic stimulation of all 4 nerves in all conditions (Figure 5). Stimulation of the sympathetic right stellate $(89 \pm 6$ vs $49 \pm 4$, $P=0)$ and parasympathetic RVST ( $34 \pm 3$ vs $51 \pm 4$, $P=.011)$ and LVST $(69 \pm 4$ vs $126 \pm 16, P=.009)$ after PVI produced significantly different changes in area from those after stimulation alone. After the CM procedure, significant changes in area from those after PVI occurred with sympathetic $\operatorname{RVST}(89 \pm 8$ vs $133 \pm 18, P=.035)$ and right stellate $(49 \pm 4$ vs $79 \pm 10, P=.023$ ) stimulation, as well as parasympathetic right stellate stimulation $(29 \pm 1$ vs $39 \pm 4, P=.045)$.

Within the left atrium, parasympathetic stimulation of the right stellate in all conditions showed no significant difference from control values (Figure 5). Likewise, after the CM procedure, sympathetic stimulation of the right stellate $(25 \pm 3$ vs $38 \pm 7, P=.422)$ and parasympathetic stimulation of the left stellate $(18 \pm 2$ vs $24 \pm 3, P=.177)$ showed no significant difference from control values. After PVI, changes in area were significantly different from those after sympathetic LVST stimulation alone $(59 \pm 4$ vs $103 \pm 11, P=$ .004). Stimulation of the sympathetic left stellate $(150 \pm 16$ vs $53 \pm 4, P=0)$ and parasympathetic LVST $(103 \pm 11$ vs $71 \pm 10, P=.013)$ after the CM procedure were significantly different from changes after PVI. 

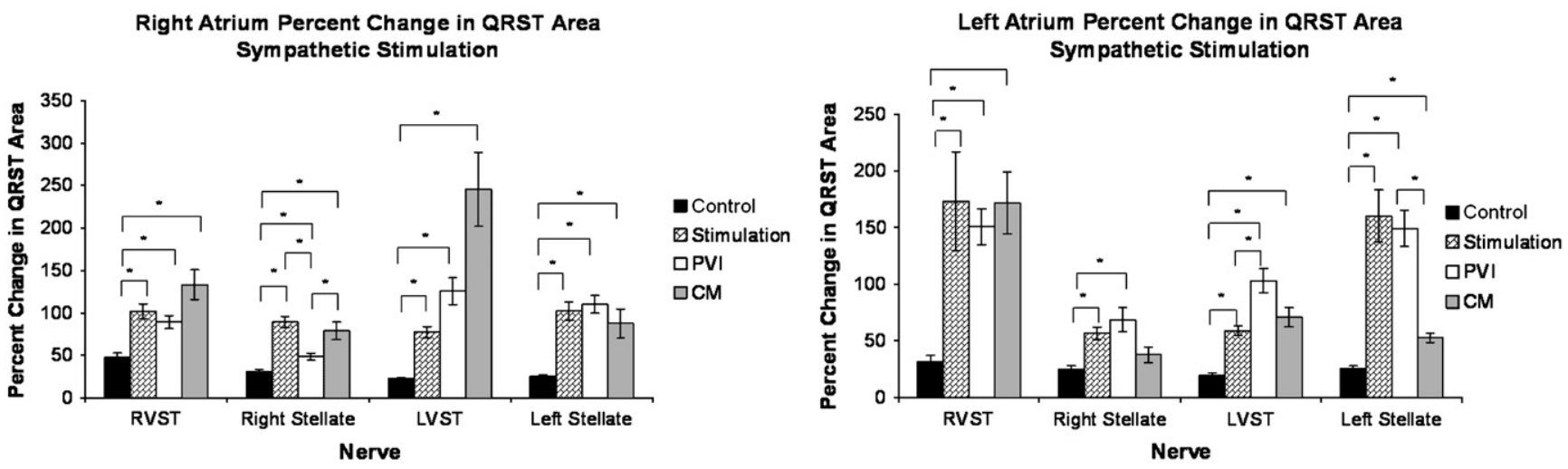

Right Atrium Percent Change in QRST Area Parasympathetic Stimulation
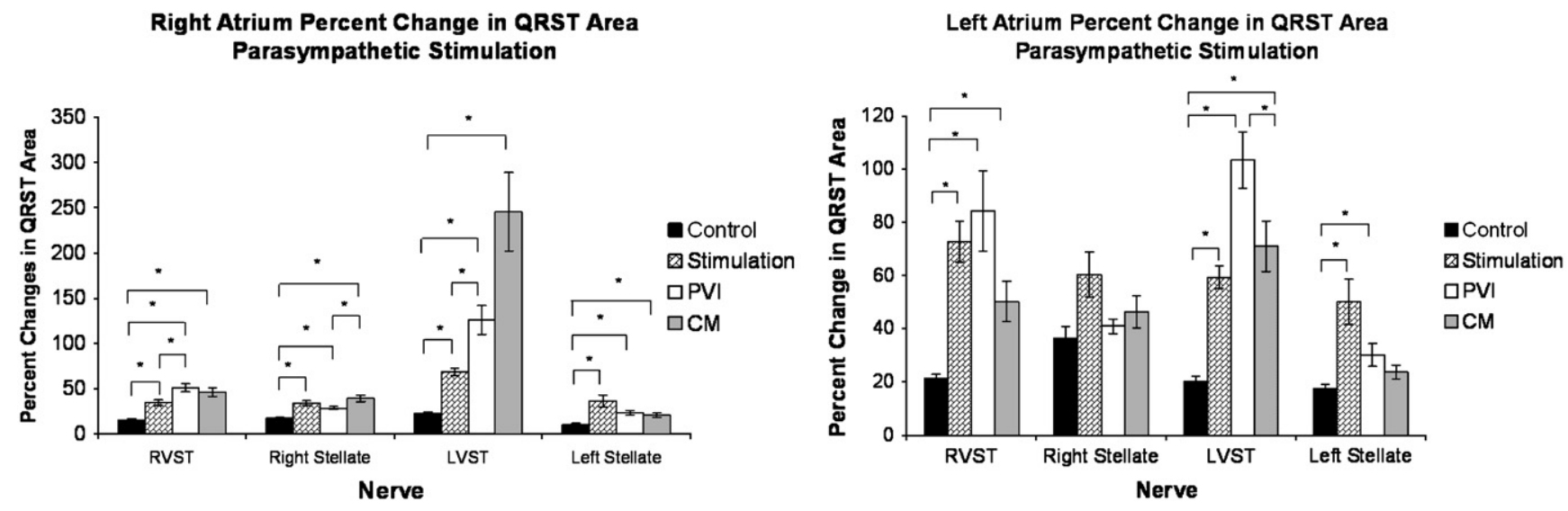

Figure 5. Right and left atrial percentage change in QRST area. ${ }^{*} P \leq 0.05 . R V S T$, Right vagosympathetic trunk; $L V S T$, left vagosympathetic trunk; PVI, pulmonary vein isolation; CM, Cox maze procedure.

The mean area changes within the right atrium were compared with those in the left atrium. With sympathetic stimulation, there was a significant difference between the right and left atria with RVST stimulation alone and PVI; right stellate stimulation alone, PVI, and the CM procedure; LVST stimulation alone and the CM procedure; and left stellate stimulation alone and PVI (Figure 6). With parasympathetic stimulation, there was a significant difference between the right and left atria with RVST stimulation alone and PVI, right stellate stimulation alone and PVI, and LVST with the CM procedure.

\section{Discussion}

The major finding of this study is that the use of bipolar radiofrequency energy to create lesions used in PVI and the CM IV procedure only partially acutely denervate those atria. In our study autonomic innervation affecting heart rate was disrupted by RFA lines, specifically in the right stellate and LVST with sympathetic stimulation and the LVST with parasympathetic stimulation. However, the effects on heart rate from RVST parasympathetic stimulation remained even after PVI and the CM procedure. This is contrary to what was shown by Do and colleagues ${ }^{15}$ with the cut-and-sew maze procedure.
They showed that stimulation of the RVST and LVST produced bradycardia before the CM procedure in acute canines. Despite the rich innervation of the right atrium, after the CM procedure, there was no effect on heart rate with stimulation. Thus the cut-and-sew CM procedure eliminated the effect on heart rate caused by vagosympathetic trunk stimulation.

Sympathetic and parasympathetic innervation affecting the AV node was not significantly disrupted by RFS lines. Few data exist on the effect of the cut-and-sew maze procedure on the AV interval. Clinical studies suggest there is little effect on AV nodal function. ${ }^{23}$

Of the 6 nerves that had significant effects on heart rate, PVI reduced the sympathetic and parasympathetic effects of the LVST. The addition of the remaining CM lesions reduced the effect of sympathetic stimulation of the right stellate. However, with the RVST (sympathetic and parasympathetic) and left stellate (sympathetic) stimulation, heart rate effects remained even after the CM lesion set. This clearly shows that although PVI partially denervates the sinus node and the CM eliminated more, there was still a significant amount of autonomic innervation after use of bipolar RFA to complete the biatrial CM procedure. 

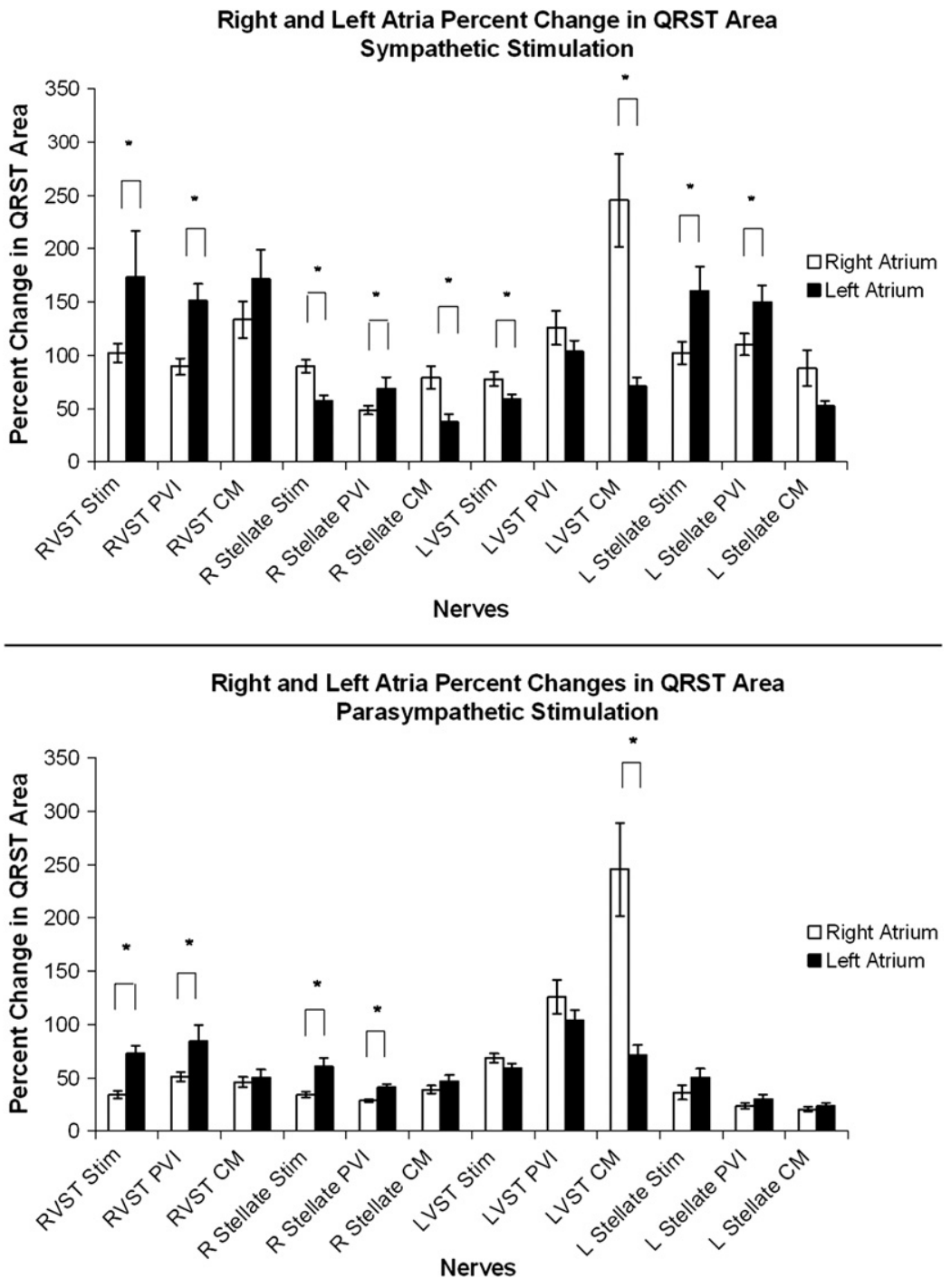

Figure 6. Right versus left atrial percentage change in QRST area. ${ }^{*} P \leq 0.05$. $R$, Right; $L$, left; RVST, right vagosympathetic trunk; LVST, left vagosympathetic trunk; Stim, stimulation; PVI, pulmonary vein isolation; $C M$, Cox maze procedure.
Geis and associates ${ }^{8}$ demonstrated the course and distribution of canine autonomic nerves to the sinus node and atria. The sinus nodal innervation was along the superior vena cava, interatrial groove, around the great arteries, and the superior left atrium. Autonomic innervation to the atrium was along the superior vena cava, interatrial groove, great arteries, superior left atrium, and inferior vena cava-inferior left atrium junction. Although the lesion set could likely disrupt many of these paths based on anatomy, nerves entering through the great arteries or various superior and inferior portions of the left atrium might not be disrupted.

In the remaining atrial myocardium, even after the CM procedure, parasympathetic and sympathetic stimulation produced changes in QRST area significantly different from control values, suggesting that the RFA biatrial CM procedure did not eliminate innervation to the atria as a whole. After PVI, changes different from those after stimulation alone were seen in $50 \%$ of stimulated nerves. However, after the CM procedure, changes different from those after PVI were seen in a different $50 \%$ of stimulated nerves. These data suggest that PVI and the $\mathrm{CM}$ procedure change the innervation to the atria but do not necessarily eliminate the autonomic innervation.

The variations found in the changes in QRST area likely reflect the fact that there is a rich plexus of nerves innervating the atrium. Pauza and coworkers ${ }^{17}$ identified 13 locations between the canine ascending aorta and pulmonary trunk, around the pulmonary veins, and on every side of the superior vena cava through which mediastinal cardiac nerves accessed the heart. It was estimated that 80,000 intrinsic neurons were associated with canine atria. Considering just the right atrium and innervation of the sinus node, it was shown that the epicardiac plexus on the root of the superior vena cava contained 456 ganglia, in which on average 54,000 nerve cells reside. $^{24}$ 
Separate analysis of the right and left atria revealed changes in QRST area significantly different from control values in all conditions for the right atrium, suggesting that even after the CM procedure, autonomic stimulation had an effect. The left atrium showed that the CM procedure eliminated right stellate sympathetic and left stellate parasympathetic effects because there was no difference from control values in the change in QRST area in these 2 nerves after the CM procedure. Although it appears that the CM procedure can eliminate some innervation to the left atrium, the same is not true for the right atrium. Within the right atrium after PVI, stimulation of 3 of 8 nerves produced changes significantly different from those produced by stimulation alone. After the $\mathrm{CM}$ procedure, there was a significant change from PVI in 3 of 8 nerves. Within the left atrium after PVI, only sympathetic stimulation of the LVST produced changes significantly different from those produced with stimulation alone. After the $\mathrm{CM}$ procedure, there was a significant change from PVI in 2 of 8 nerves. This further suggests that both PVI and the CM procedure change the innervation to the right and left atria.

Both PVI and the CM procedure only partially denervate the atria. It is unclear whether this denervation would eliminate any potential arrhythmogenic influences. In fact, partial denervation can be arrhythmogenic by increasing the inhomogeneity of refractory period. ${ }^{25}$ If future studies support the present data suggesting that vagal innervation is an important arrhythmogenic mechanism for the development or maintenance of AF, surgical treatment might have to include additional lesions to completely denervate the atria. Some investigators now are ablating the ganglionated plexus as an adjunctive therapy to eliminate vagal inputs to the atria. ${ }^{26}$ Beyond the acute denervation, studies will be needed to determine whether the denervation persists. Both in animal studies and in human subjects, reinnervation occurs over time after the CM procedure. ${ }^{12,27}$

\section{References}

1. Cox JL, Schuessler RB, Lappas DG, Boineau JP. An 8 1/2-year clinical experience with surgery for atrial fibrillation. Ann Surg. 1996;224:267-73.

2. Prasad SM, Maniar HS, Camillo CJ, Schuessler RB, Boineau JP, Sundt TM 3rd, et al. The Cox maze III procedure for atrial fibrillation: long-term efficacy in patients undergoing lone versus concomitant procedures. J Thorac Cardiovasc Surg. 2003;126:1822-8.

3. Gaynor SL, Diodato MD, Prasad SM, Ishii Y, Schuessler RB, Bailey MS, et al. A prospective, single-center clinical trial of a modified Cox maze procedure with bipolar radiofrequency ablation. $J$ Thorac Cardiovasc Surg. 2004;128:535-42.

4. Lall SC, Melby SJ, Voeller RK, Zierer A, Bailey MS, Guthrie TJ, et al. The effect of ablation technology on surgical outcomes after the Coxmaze procedure: a propensity analysis. $J$ Thorac Cardiovasc Surg. 2007:133:389-96.

5. Wolf RK, Schneeberger EW, Osterday R, Miller D, Merrill W, Flege JB Jr, et al. Video-assisted bilateral pulmonary vein isolation and left atrial appendage exclusion for atrial fibrillation. $J$ Thorac Cardiovasc Surg. 2005;130:797-802.

6. Sagbas E, Akpinar B, Sanisoglu I, Caynak B, Tamtekin B, Oral K, et al. Video-assisted bilateral epicardial pulmonary vein isolation for the treatment of lone atrial fibrillation. Ann Thorac Surg. 2007;83:1724-30.
7. Schuessler RB, Boineau JP, Wylds AC, Hill DA, Miller CB, Roeske WR. Effect of canine cardiac nerves on heart rate, rhythm, and pacemaker location. Am J Physiol Heart Circ Physiol. 1986;250: H630-44.

8. Geis WP, Kaye MP, Randall WC. Major autonomic pathways to the atria and S-A and A-V nodes of the canine heart. Am J Physiol. 1973;224: 202-8.

9. Zipes DP, Mihalick MJ, Robbins GT. Effects of selective vagal and stellate ganglion stimulation of atrial refractoriness. Cardiovasc Res. 1974;8:647-55.

10. Chen PS, Tan AY. Autonomic nerve activity and atrial fibrillation. Heart Rhythm. 2007;4(suppl):S61-4.

11. Hou Y, Scherlag BJ, Lin J, Zhang Y, Lu Z, Truong K, et al. Ganglionated plexi modulate extrinsic cardiac autonomic nerve input: effects on sinus rate, atrioventricular conduction, refractoriness, and inducibility of atrial fibrillation. J Am Coll Cardiol. 2007;50:61-8.

12. Mabuchi M, Imamura M, Kubo N, Morita K, Noriyasu K, Tsukamoto T, et al. Sympathetic denervation and reinnervation after the maze procedure. J Nucl Med. 2005;46:1089-94.

13. Pasic M, Musci M, Siniawski H, Grauhan O, Edelmann B, Tedoriya T, et al. The Cox maze iii procedure: parallel normalization of sinus node dysfunction, improvement of atrial function, and recovery of the cardiac autonomic nervous system. J Thorac Cardiovasc Surg. 1999;118: 287-95.

14. Vogt PR, Brunner La Rocca HP, Candinas R, Gasser J, Zund G, Schonbeck M, et al. Temporary loss of cardiac autonomic innervation after the maze procedure. Eur J Cardiothorac Surg. 1997;12:75-81.

15. Do O-BP, Dandan N, Cradinal R. Effects of maze III procedure on atrial parasympathetic innervation. Can J Cardiol. 1996;12:145E.

16. Lonnerholm S, Blomstrom P, Nilsson L, Ericson M, Kesek M, Jideus L, et al. Autonomic denervation after the Maze procedure. Pacing Clin Electrophysiol. 2003;26:587-92.

17. Pauza DH, Skripka V, Pauziene N. Morphology of the intrinsic cardiac nervous system in the dog: a whole-mount study employing histochemical staining with acetylcholinesterase. Cells Tissues Organs. 2002;172: 297-320.

18. Pauza DH, Skripka V, Pauziene N, Stropus R. Morphology, distribution, and variability of the epicardiac neural ganglionated subplexuses in the human heart. Anat Rec. 2000;259:353-82.

19. Melby SJ, Gaynor SL, Lubahn JG, Lee AM, Rahgozar P, Caruthers SD, et al. Efficacy and safety of right and left atrial ablations on the beating heart with irrigated bipolar radiofrequency energy: a long-term animal study. J Thorac Cardiovasc Surg. 2006;132:853-60.

20. Page PL, Dandan N, Savard P, Nadeau R, Armour JA, Cardinal R. Regional distribution of atrial electrical changes induced by stimulation of extracardiac and intracardiac neural elements. J Thorac Cardiovasc Surg. 1995;109:377-88.

21. Page PCR. Efferent autonomic innervation of the atrium: assessment by isointegral mapping. In: Shenase MB, Breithardt G, eds. Cardiac mapping. 2nd ed. Elmsford (NY): Blackwell Publishing; 2003. p. 363-71.

22. Wilson FN, Macleod AG, Barker PS, Johnston FD. The determination and the significance of the areas of the ventricular deflections of the electrocardiogram. Am Heart J. 1934;10:46-61.

23. Albage A, Lindblom D, Insulander P, Kenneback G. Electrophysiological evaluation of the sinus node and the cardiac conduction system following the maze procedure for atrial fibrillation. Pacing Clin Electrophysiol. 2004;27:194-203.

24. Pauza DH, Skripka V, Pauziene N, Stropus R. Anatomical study of the neural ganglionated plexus in the canine right atrium: implications for selective denervation and electrophysiology of the sinoatrial node in dog. Anat Rec. 1999;255:271-94.

25. Hirose M, Leatmanoratn Z, Laurita KR, Carlson MD. Partial vagal denervation increases vulnerability to vagally induced atrial fibrillation. J Cardiovasc Electrophysiol. 2002;13:1272-9.

26. Mehall JR, Kohut RM Jr, Schneeberger EW, Taketani T, Merrill WH, Wolf RK. Intraoperative epicardial electrophysiologic mapping and isolation of autonomic ganglionic plexi. Ann Thorac Surg. 2007;83:538-41.

27. Oh S, Zhang Y, Bibevski S, Marrouche NF, Natale A, Mazgalev TN. Vagal denervation and atrial fibrillation inducibility: epicardial fat pad ablation does not have long-term effects. Heart Rhythm. 2006;3:701-8. 